\title{
Gremlin Promotes Peritoneal Membrane Injury in an Experimental Mouse Model and Is Associated with Increased Solute Transport in Peritoneal Dialysis Patients
}

\author{
Imad Siddique, ${ }^{*}$ Simon P. Curran, ${ }^{\dagger}$ Ayesha Ghayur, ${ }^{*}$ Limin Liu, ${ }^{*}$ Wei Shi, ${ }^{\ddagger}$ Catherine M. Hoff, ${ }^{\S}$ Azim S. Gangji, * \\ K. Scott Brimble, ${ }^{*}$ and Peter J. Margetts*
}

\begin{abstract}
From the Department of Medicine, ${ }^{*}$ McMaster University, Hamilton, Ontario, Canada; the Division of Nephrology, ${ }^{\dagger}$ University of Toronto, Toronto, Ontario, Canada; the Children’s Hospital Los Angeles, ${ }^{\ddagger}$ University of Southern California, Los Angeles, California; and the Renal Division, ${ }^{\S}$ Baxter Healthcare, McGaw Park, Illinois
\end{abstract}

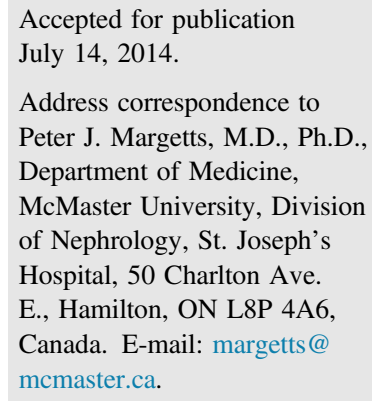

\begin{abstract}
The peritoneal membrane becomes damaged in patients on peritoneal dialysis (PD). Gremlin 1 (GREM1) inhibits bone morphogenic proteins (BMPs) and plays a role in kidney development and fibrosis. We evaluated the role of gremlin in peritoneal fibrosis and angiogenesis. In a cohort of 32 stable PD patients, GREM1 concentration in the peritoneal effluent correlated with measures of peritoneal membrane damage. AdGrem1, an adenovirus to overexpress gremlin in the mouse peritoneum, induced submesothelial thickening, fibrosis, and angiogenesis in $\mathrm{C} 57 \mathrm{BL} / 6$ mice, which was associated with decreased expression of BMP4 and BMP7. There was evidence of mesothelial cell transition to a mesenchymal phenotype with increased $\alpha$ smooth muscle actin expression and suppression of E-cadherin. Some of the GREM1 effects may be reversed with recombinant BMP7 or a pan-specific transforming growth factor $\beta$ (TGF- $\beta$ ) antibody. Neovascularization was not inhibited with a TGF- $\beta$ antibody, suggesting a TGF- $\beta$-independent angiogenic mechanism. Swiss/Jackson Laboratory $(\mathrm{SJL})$ mice, which are resistant to TGF- $\beta$-induced peritoneal fibrosis, responded in a similar fashion to AdGrem 1 as did $\mathrm{C} 57 \mathrm{BL} / 6$ mice with fibrosis, angiogenesis, and mesothelial-to-mesenchymal transition. GREM1 was associated with up-regulated TGF- $\beta$ expression in both SJL and C57BL/ 6 mice, but SJL mice demonstrated a defective TGF- $\beta$-induced GREM1 expression. In summary, GREM1 induces fibrosis and angiogenesis in mouse peritoneum and is associated with increased solute transport in these PD patients. (Am J Pathol 2014, 184: 2976-2984; http://dx.doi.org/10.1016/ j.ajpath.2014.07.018)
\end{abstract}

Peritoneal dialysis (PD) is an effective form of renal replacement therapy, with similar survival ${ }^{1,2}$ and quality of life ${ }^{3}$ compared with hemodialysis, but with decreased costs. ${ }^{4}$ PD treatment, and the associated peritoneal membrane glucose exposure, leads to peritoneal fibrosis and submesothelial thickening. ${ }^{5}$ This fibrosis is associated with angiogenesis and decreased osmotic conductance, leading to ultrafiltration dysfunction. ${ }^{6}$ Angiogenesis leads to increased solute transport, which has been associated with increased mortality and technique failure.

In response to $\mathrm{PD}$, the epithelial-like mesothelial cells that line the peritoneal cavity undergo a transition from an epithelial phenotype to a mesenchymal phenotype. ${ }^{8}$ Transitioned mesothelial cells produce vascular endothelial growth factor
$\left(\mathrm{VEGF}^{9}\right.$ and are associated with increased peritoneal membrane solute transport. ${ }^{10}$ Transforming growth factor $\beta$ (TGF- $\beta$ ) has been demonstrated to induce epithelial mesenchymal transition (EMT) in mesothelial cells, ${ }^{11}$ whereas bone morphogenic protein (BMP) 7 has been shown to be a protective factor that maintains the epithelial phenotype of mesothelial cells. ${ }^{12}$

Supported by the Renal Division, Baxter Healthcare; Kidney Foundation of Canada grant KFOC130020 (P.M.); and St. Joseph's Healthcare, Hamilton, Ontario, Canada.

A portion of this work was presented at the American Society of Nephrology Kidney Week 2011, November 8-13, 2011, Philadelphia, PA.

Disclosures: C.M.H. is an employee of the Renal Division, Baxter Healthcare. The FLUID study was supported by an extramural grant from Baxter Healthcare. 
Gremlin 1 (GREM1) is a small secreted glycoprotein originally identified because of its role in kidney development. ${ }^{13}$ The main action of GREM1 is to bind and inactivate BMP2, BMP4, and BMP7. ${ }^{14,15}$ These BMPs are widely expressed and are involved in skeletal repair and regeneration. ${ }^{16}$ GREM1 is important in organogenesis and regulates branching morphogenesis in the lung ${ }^{14}$ and kidney. ${ }^{17}$ Furthermore, by promoting EMT in adult organs, gremlin has been shown to play a role in lung ${ }^{18}$ and kidney fibrosis. ${ }^{19}$ Recent studies have also identified a role for gremlin in angiogenesis through noncanonical binding to VEGF receptor $2 .^{20,21}$

The role of gremlin in peritoneal membrane injury in patients on PD has not been previously described. We measured gremlin protein concentration in the PD effluent of patients and evaluated the effect of gremlin on the peritoneal membrane in two different strains of mice.

\section{Materials and Methods}

\section{Patient Samples}

Thirty-two stable PD patients who were recruited as part of an ongoing randomized trial of bioimpedance-directed ultrafiltration therapy [FLUID study (Effect of Bio-impedance Analysis and Vitamin D Versus Usual Care on Left Ventricular Mass in Peritoneal Dialysis Patients: A Randomized Controlled Trial) ${ }^{22}$ ], provided an overnight PD effluent and results of a daytime peritoneum equilibrium test (PET). These patients had been peritonitis free for at least 3 months before samples were taken. The PET was performed as previously described, with a 4-hour dwell of $4.25 \%$ glucose solution. ${ }^{23}$

We used two methods to evaluate GREM1 concentration in PD effluent. For 25 samples, equal volumes of PD fluid were loaded on and SDS-PAGE gel, and densitometry analysis was performed to quantify GREM1 in the PD effluent (Santa Cruz Biotechnology, Inc., Dallas, TX). We used a nonspecific band from Coomassie Blue-stained blots for loading control. In 21 patients with serum samples available, we performed an enzyme-linked immunosorbent assay (ELISA) for GREM1 on both PD and serum (Elabscience Biotechnology Company, Ltd., Wuhan, China). We compared the effluent concentration of GREM1 with the dialysate/plasma (D/P) creatinine and the 4-hour to initial glucose. We also compared dialysate/serum values of GREM1. Further, we compared the quantified PD concentration of GREM1 and other clinical features such as age, time on PD, and diabetes status.

\section{Animal Studies}

C57BL/6 (B6) mice ( $n=5$ or 6 mice per group at each time point) were infected with an adenovirus expressing GREM1 (AdGrem1) at a dose of $3 \times 10^{8}$ plaque-forming units (PFU). All adenoviruses were diluted in $100 \mu \mathrm{L}$ of saline and administered by i.p. injection. Animals were euthanized 7 , 14, or 21 days after infection. In mice exposed to AdGrem1, we treated one group with 1D11 (a pan-specific TGF- $\beta$ inhibitor provided by Genzyme Corp., Framingham, MA) at a dose of $10 \mathrm{mg} / \mathrm{kg}^{24}$ A second group of mice exposed to AdGrem1 also received recombinant BMP7 (rBMP7) (Creative Biomart, Shirley, NY) at a dose of 300 $\mu \mathrm{g} / \mathrm{kg} .{ }^{25}$ Both 1D11 and rBMP7 were delivered on days 1, 3 , and 5 post-infection with AdGrem1. Mice in these two treatment groups were euthanized on day 7.

In a second experiment, Swiss/Jackson Laboratory (SJL) or B6 mice ( $n=5$ or 6 per group) were infected with AdGrem1 or control adenovirus (AdDL) at a dose of $3 \times 10^{8}$ PFU. In a third experiment, SJL or B6 mice were administered an adenovirus-expressing active TGF- $\beta 1$ (AdTGFB1) at a dose of $1 \times 10^{8} \mathrm{PFU}$ i.p. Animals in the second and third experiments were sacrificed 7 days after infection.

All mice were 5 to 6 weeks old at infection. A nonexpressing adenovirus (AdDL) was used at the same dose as was the active virus in all experiments, to control for viral effects. The Greml gene used in the construction of AdGREM1 was derived from mouse tissue. ${ }^{26}$ The $T g f b l$ gene used in AdTGFB1 was derived from porcine tissue and was modified to express the active form of TGF- $\beta 1{ }^{27} \mathrm{Ad}-$ enoviruses were expanded, purified, and measured as previously described. ${ }^{28}$

\section{Histological Examination}

At the time of euthanasia, the anterior abdominal wall was resected and the upper portion of this tissue was formalin fixed. Sections were stained for von Willebrand factor-related antigen (Dako North America, Inc., Carpenteria, CA) as previously described. ${ }^{29}$ Eight to 10 images were taken per slide and analyzed by image-analysis software (Northern Eclipse version 6.0; Empix Imaging Inc., Mississauga, ON, Canada). Vascular density was measured as the number of vessels within $500 \mu \mathrm{m}$ of the superficial mesothelial cell layer. For immunofluorescence, sections were stained for $\alpha$ smooth muscle actin (Dako), followed by a secondary rabbit antimouse antibody labeled with Texas Red (Molecular Probes, Eugene, OR) and fluorescein isothiocyanate-conjugated anticytokeratin (Sigma-Aldrich Canada Company, Oakville, ON, Canada). Dual-labeled cells were evaluated in a blinded fashion by one of the investigators (I.S.) from five high-power fields from each section. Dual-labeled cells were identified either within the upper mesothelial cell layer (noninvading) or within the submesothelium (invading).

\section{Western Blot Analysis}

Protein was extracted from frozen omental tissue and analyzed by Western blot for BMP4 (R\&D Systems Inc., Minneapolis, MN), BMP7 (Abcam plc, Toronto, ON, Canada), gremlin (Cell Signaling Technology, Inc., Whitby, ON, Canada), and E-cadherin (BD Biosciences, Mississauga, 
ON, Canada). Band density was quantified and normalized to $\alpha$-tubulin (Sigma-Aldrich).

\section{RNA Analysis}

Quantitative real-time PCR for Vegf, $\alpha 2$ chain of type 1 collagen (Coll $a 2), T g f b$, and E-cadherin $(C d h l)$ was performed using ABI Prism 7500 Sequence Detector (Applied Biosystems Inc., Foster City, CA). RNA was extracted from anterior abdominal wall tissue as has been previously described. ${ }^{29}$ Gene expression was normalized to both Gapdh and $18 s$, with similar results. Results normalized to Gapdh are shown.

\section{Statistical Analysis}

All data are expressed as means \pm SD and were first analyzed for normal distribution using the Kolmogorov-Smirnov test. Those data that were normally distributed were analyzed by one-way analysis of variance with Tukey post hoc correction (between groups) or by $t$-test. Nonparametric data were analyzed using the Kruskal-Wallis test. Correlation with solute transport was made with Spearman rank correlation (IBM SPSS Statistics, Armonk, NY).

\section{Results}

\section{Association of GREM1 Effluent Concentration with Peritoneal Membrane Solute Transport in PD Patients}

We gathered overnight PD effluent from 32 stable PD patients. The mean \pm SD age of these patients was $62.5 \pm 12$ years, the mean duration of PD was $673 \pm 599$ days. Twelve of the patients (38\%) had diabetes.

We found that GREM1 protein was abundantly expressed in the overnight PD effluent in the patients, as measured by Western blot analysis (Figure 1A). There was a significant correlation between GREM1 concentration in PD effluent and measures of peritoneal membrane solute transport, including 4-hour $\mathrm{D} / \mathrm{P}$ creatinine $(r=0.472, P=0.011)$ and initial glucose $(r=-0.597, P=0.001)$ (Figure 1, B and C). We repeated this in a cohort of 21 stable PD patients using ELISA. In this group, we were able to measure both serum and PD effluent GREM1. We confirmed that PD effluent GREM1 was correlated with D/P creatinine (Figure 1D). There was no correlation between dialysate and serum GREM1 $(R=-0.172, P=0.455)$ (Figure 1E). Furthermore, dialysate concentrations of GREM1 were significantly higher than were the serum concentrations in all samples tested $(P<0.001)$. On average, D/P GREM1 was 3.9 (range, 1.03 to 10.34), suggesting an almost fourfold increase in dialysate GREM1 compared with that in serum.

Patients with diabetes had a significantly higher PD effluent GREM1 concentration compared with those without (3.03 versus $2.44 \mathrm{ng} / \mathrm{mL}, P=0.011$ ). There was no correlation between GREM1 PD concentration and time on dialysis or age.

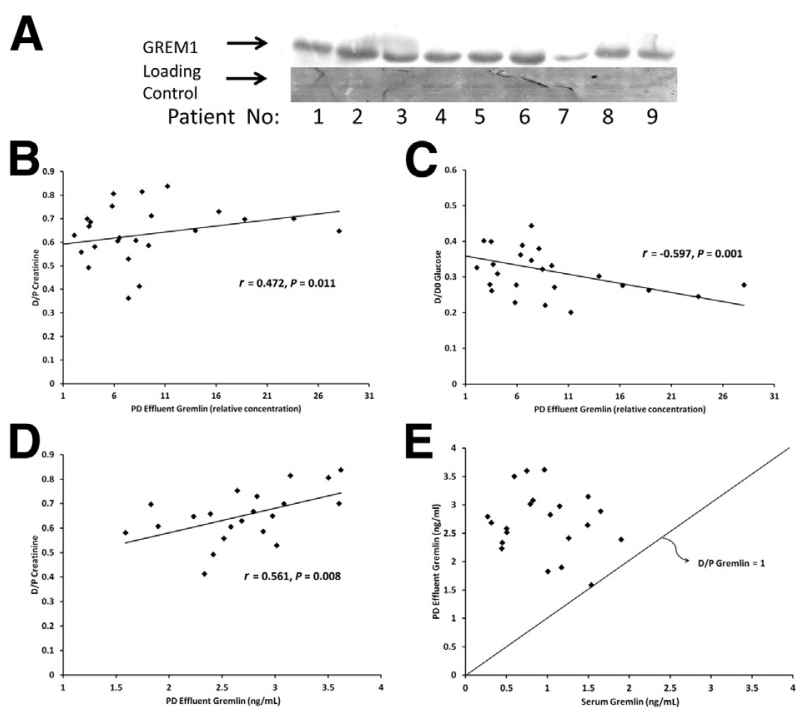

Figure 1 Overnight peritoneal effluent was obtained from 32 stable peritoneal dialysis (PD) patients. A: Equal volume of peritoneal fluid analyzed by Western blot; band density normalized using a nonspecific band on Coomassie Blue-stained blots. B and C: GREM1 concentration correlates with D/P creatinine (B) and inversely with 4 hours to initial (D/DO) glucose (C). D: These results were confirmed in a second cohort of 21 stable peritoneal dialysis patients using ELISA. PD effluent GREM1 correlates with D/P creatinine. E: Serum GREM1 levels in these patients. There is no correlation between serum and dialysate concentration of GREM1; dialysate GREM1 concentrations are higher than serum concentrations in all patients (mean $\mathrm{D} / \mathrm{P}$ GREM1 is 3.9, line indicating D/P gremlin $=1) \cdot n=25(\mathbf{A}) \cdot r=$ $0.472, P=0.011(\mathrm{~B}) ; r=-0.597, P=0.001(\mathrm{C}) ; r=0.561, P=0.008$ (D); $(r=-0.172, P=0.455)(\mathrm{E})$.

Effects of Gremlin on Submesothelial Thickening and Angiogenesis in C57BL/6 (B6) Mice

As we have previously described, ${ }^{30}$ AdDL had no visible effects on the peritoneal tissues (Figure 2A). Submesothelial thickening, cellular proliferation, and fibrosis in animals exposed to AdGrem1 were maximal 7 days after, but persisted to 21 days after, adenoviral infection (Figure 2, $\mathrm{B}-\mathrm{D}$ and $\mathrm{G})$. Angiogenesis was also significantly increased after AdGrem1 infection and resolved by 14 days after infection (Figure 2H).

Two additional groups of B6 mice were treated for 1 week with AdGrem1 and rBMP7 (Figure 2E), or AdGrem1 and 1D11 (Figure 2F), a pan-TGF- $\beta$ inhibitory antibody. ${ }^{31}$ Treatment with rBMP7 had no significant effect on the peritoneal membrane on histological examination, whereas 1D11 was associated with reduced submesothelial thickening (Figure 2G), without significantly affecting angiogenesis (Figure 2H).

\section{Effects of GREM1 on BMP7, BMP4, and E-Cadherin Expression}

Overexpression of GREM1 were associated with decreases in BMP7, BMP4, and E-cadherin expression (Figure 3, A-D). These effects were seen at 7 and 14 days after 

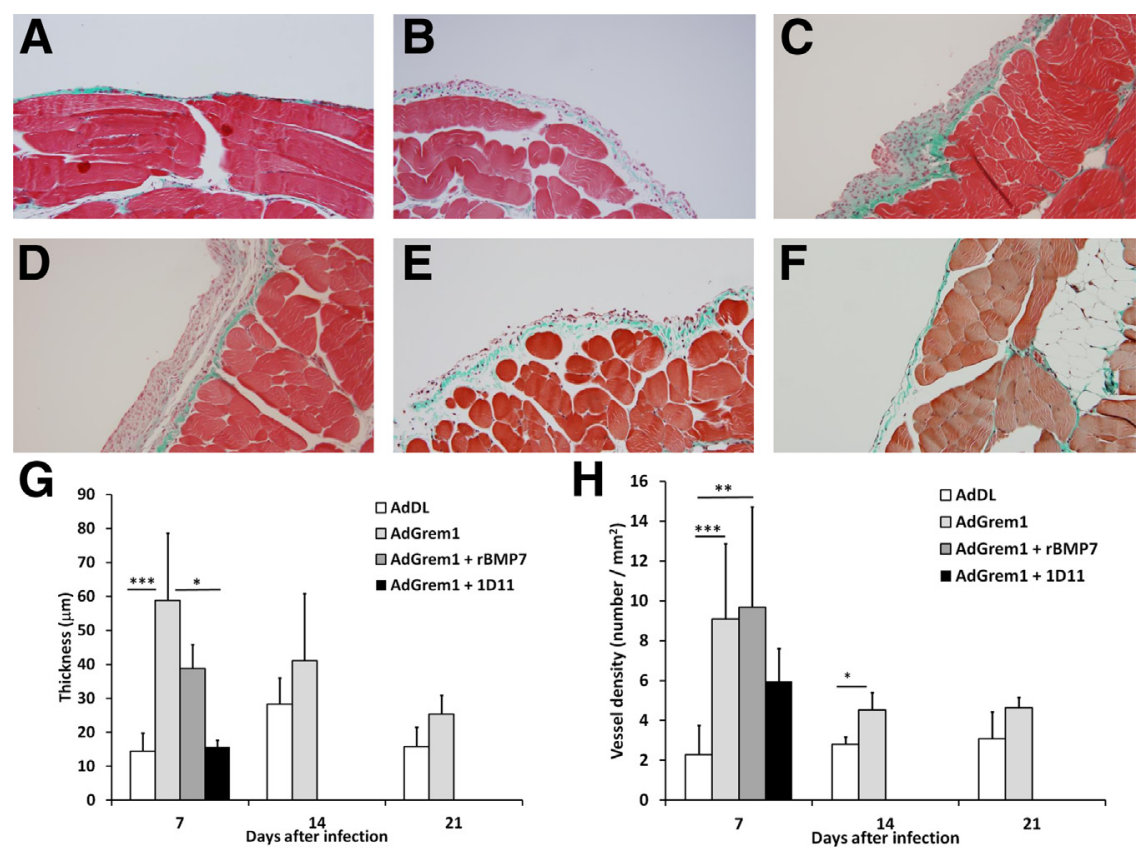

Figure 2 Histologic analysis of the anterior abdominal wall after exposure to AdDL or AdGrem1 in B6 mice. A: Normal peritoneal structure seen 7 days after AdDL. B-D: Increased submesothelial thickening and fibrosis 7, 14, and 21 days after exposure to AdGrem1. E: In animals treated with AdGrem 1 and rBMP7, there is ongoing submesothelial thickening and angiogenesis. F: There is a decrease in submesothelial thickening in animals treated with AdGrem1 and 1D11. Sections were stained with Masson's trichrome. G-H: Quantitation of submesothelial histological examination in B6 mice exposed to AdDL or AdGrem1. G: Significantly increased submesothelial thickening is seen after exposure to AdGrem. The submesothelial thickening is significantly reduced by treatment with 1D11. H: Submesothelial angiogenesis seen in the peritoneal tissues of $\mathrm{B} 6$ mice exposed to AdGrem1 is not prevented by treatment with rBMP7 or 1D11. $n=4$ for control (AdDL) group; $n=6$ for treatment groups at each time point. Data are expressed as means $\pm \mathrm{SD}(\mathbf{G}$ and $\mathbf{H})$. ${ }^{*} P<0.05$, $* * P<0.01$, and ${ }^{* * *} P<0.001$. Original magnification: $\times 100(\mathbf{A}-\mathbf{F})$. infection with AdGrem1. Treatment with either rBMP7 or 1D11 appeared to have significantly reversed the GREM1induced down-regulation of BMP4, BMP7, and E-cadherin. We found that GREM1 was associated with significant increases in Colla2 gene expression in peritoneal tissues (Figure 3, E and F). Treatment with rBMP7 or 1D11 apparently significantly reversed the effect of GREM1 on Colla2 and Vegf gene expression.

\section{Effect of GREM1 on Cellular Transition}

We stained sections of the peritoneal membrane from the anterior abdominal wall with epithelial (cytokeratin) and mesenchymal ( $\alpha$ smooth muscle actin) antibodies. GREM1 appeared to have induced mesothelial cell transition, with evidence of mesothelial cells expressing both epithelial and mesenchymal markers (Figure 4). There was still

A
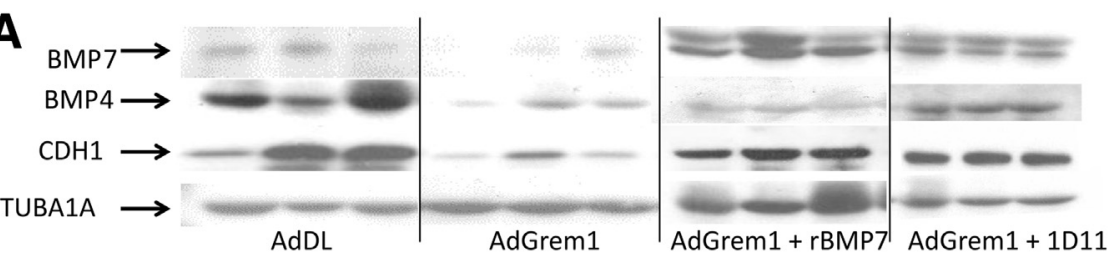

B
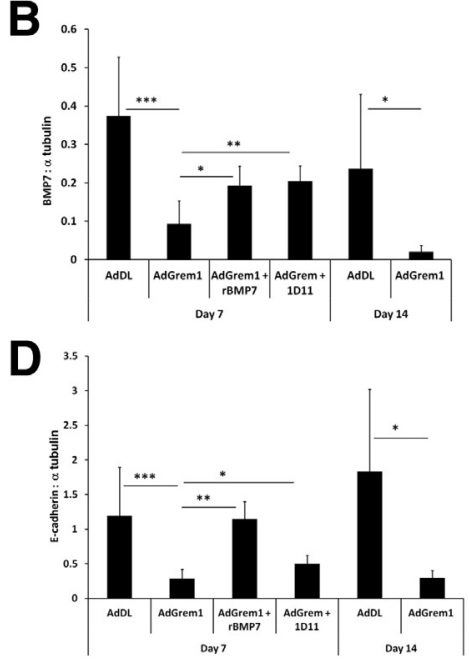

C

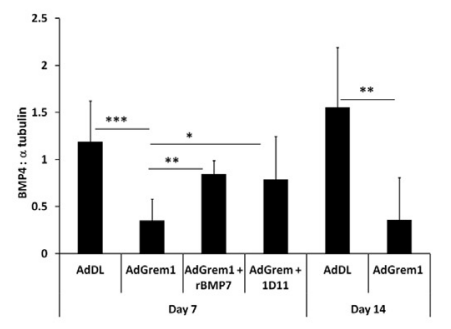

E

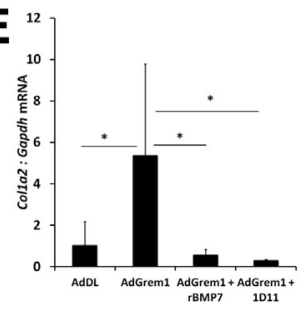

$\mathbf{F}$

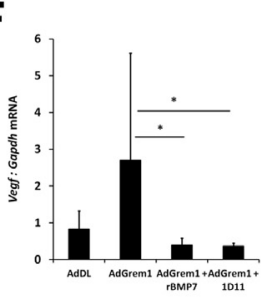

Figure 3 Adenovirus-mediated GREM1 overexpression is associated with decreased expression of BMP 7, BMP4, and E-cadherin (CDH1) in B6 mice. These effects are reversed by treatment with $\mathrm{rBMP7}$ or 1D11. A: Representative Western blot analysis. Each lane represents protein from an individual mouse. Band density was normalized to $\alpha$-tubulin (TUBA1A) and quantified in B-D. Increased gene expression of Col1a2 (E) and Vegf $(\mathbf{F})$ seen in the peritoneal tissue of animals 7 days after treatment with AdGrem1; both of these effects are reversed by rBMP7 or 1D11. $n=4$ for control (AdDL) group; $n=6$ for treatment groups at each time point. Data are expressed as means \pm SD $(\mathbf{B}-\mathbf{E}) .{ }^{*} P<0.05$, $* * P<0.01$, and $* * * P<0.001$. 

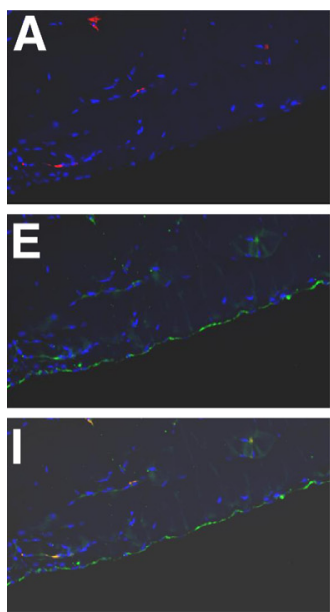

AdDL
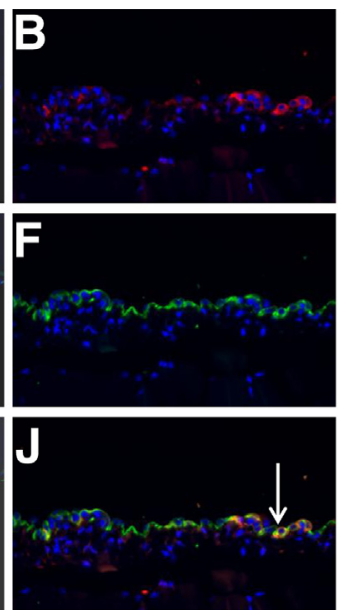

AdGrem1
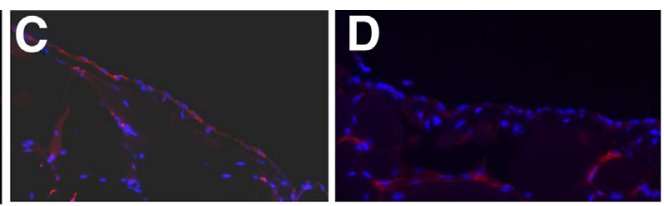

$\alpha-$

smooth

muscle

actin
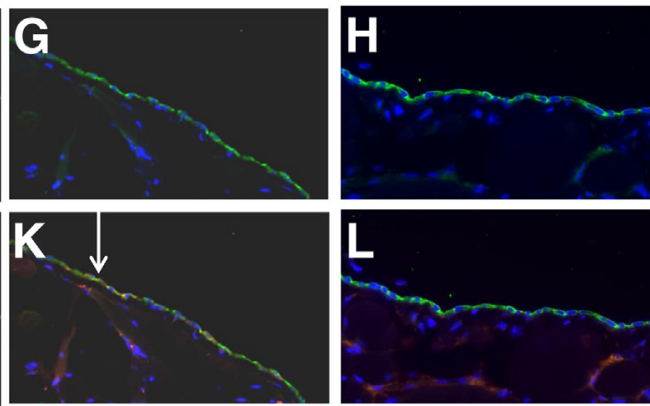

AdGrem1

+ rBMP7

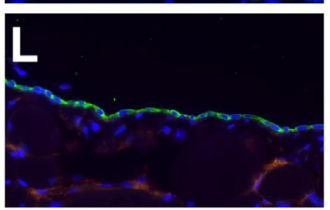

AdGrem1

$+1 \mathrm{D} 11$

M

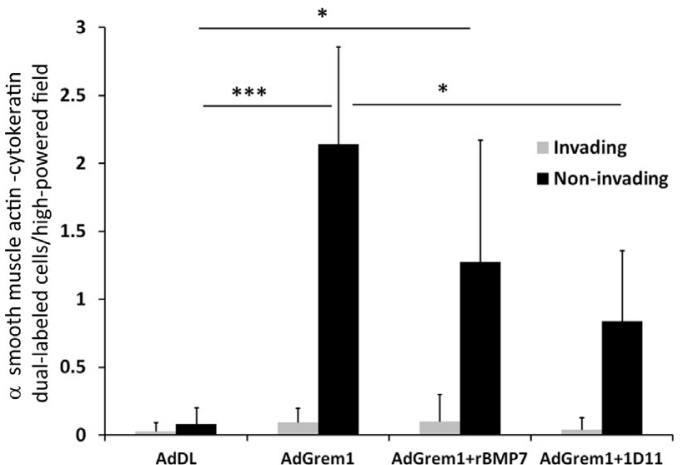

evidence of dual-labeled cells after treatment of these mice with rBMP7, but the mesothelial cell transition was abolished with 1D11 treatment. The majority of the transitioned or dual-labeled cells were found within the mesothelial cell layer (noninvading). A small number of dual-labeled cells were found in the submesothelium. These invading transitioned cells were numerically increased by threefold with AdGrem1 compared with those in AdDL-treated mice, but the difference was not statistically significant.

\section{Mouse Strain-Dependent GREM1 Effects}

We previously found that SJL mice were resistant to TGF- $\beta$-induced peritoneal fibrosis, whereas B6 mice developed significant fibrosis and angiogenesis. ${ }^{32}$ We therefore evaluated the response in SJL mice to GREM1. Surprisingly, we found that SJL mice appeared to have responded strongly to AdGrem1 (Figure 5). Seven days after infection, these mice developed a significant increase in submesothelial thickening with associated fibrosis and cellular proliferation (Figure 5, B and D). The SJL mice had an exaggerated angiogenic response compared with that in B6 mice delivered the same dose of AdGrem1 (Figure 5E).
Figure 4 Evidence of EMT in peritoneal tissue. Sections stained for $\alpha$ smooth muscle actin (red, $\mathbf{A}-\mathbf{D}$ ), cytokeratin (green, $\mathbf{E}-\mathbf{H}$ ) and merged (I-L). A, E, and I: There is no evidence of EMT after infection with AdDL. B, F, and J: B6 mice demonstrate evidence of EMT with cells labeled with both $\alpha$ smooth muscle actin and cytokeratin (arrow). C, G, and K: Similar co-expression is seen in animals treated with AdGrem1 and rBMP7 (arrow). D, H, and L: Blockade of TGF- $\beta$ using $1 \mathrm{~d} 11$ prevents EMT. Blue - DAPI nuclear stain. M: Dual-labeled cells are quantified as noninvading (within the mesothelial cell layer) and invading (in the submesothelial zone). There is a significant decrease in EMT in AdGrem1-exposed animals treated with 1D11. $n=4$ for control (AdDL) animals; $n=6$ for treatment groups. Data are expressed as means \pm SD (M). ${ }^{*} P<0.05,{ }^{* *} P<0.001$. Original magnification: $\times 200(\mathbf{A}-\mathbf{L})$.

GREM1 was associated with a significant increase in peritoneal tissue gene expression of Colla2 and Vegf in B6 mice, whereas there was no significant change in SJL mice (Figure 5, F and G). GREM1 was associated with significantly decreased Cdhl gene expression in both SJL and B6 mice (Figure 5H).

\section{Interaction between GREM1 and TGF- $\beta$}

Our data suggest that GREM1 and TGF- $\beta$ appear to have overlapping effects of fibrosis and angiogenesis in peritoneal tissues. We evaluated the impact of GREM1 overexpression on TGF- $\beta$ and found that AdGrem1 induced significant $T g f b$ gene expression in both SJL and B6 mice (Figure 6A). We then used AdTGFB and infected the peritoneum. In this experiment, AdTGFB induced a significant increase in Greml gene and protein expression in B6 mice but not in SJL mice (Figure 6, B-D).

\section{Discussion}

Exposure of the peritoneal membrane to dialysis solutions leads to injury to the peritoneal mesothelial cells. ${ }^{33}$ In the setting of 


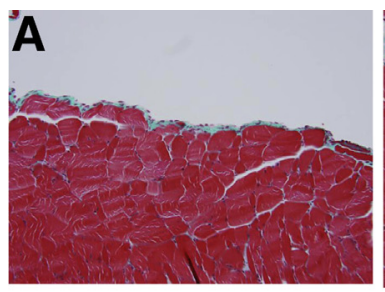

D
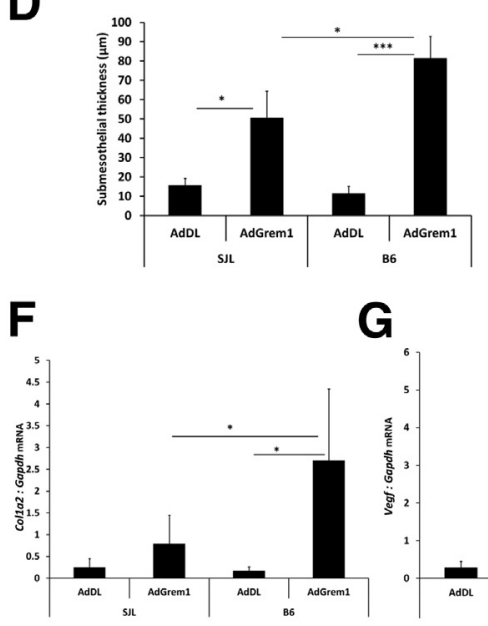

G
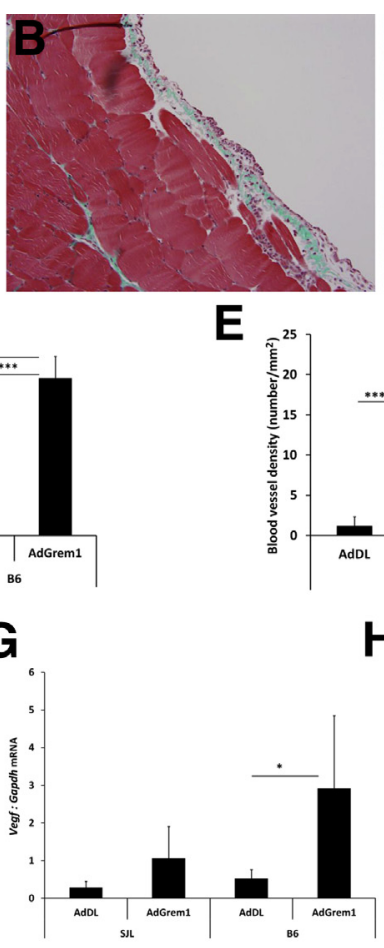

E

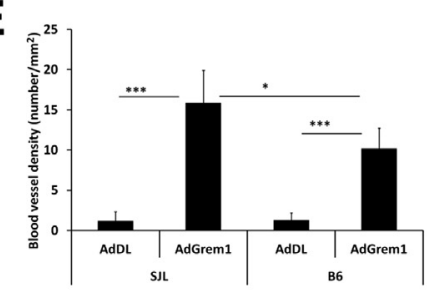

H

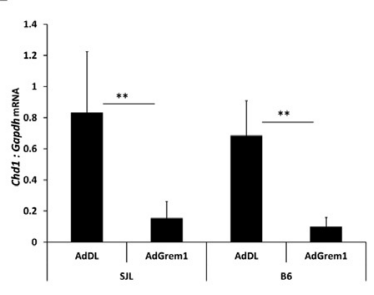

Figure 5 Effect of GREM1 on the peritoneum of SJL and B6 mice. Histological examination of the anterior abdominal wall of SJL mice 7 days after treatment with AdDL (A) or AdGrem1 (B). Sections were stained with Masson's trichrome. C: Peritoneum of B6 mice treated with AdGrem1. D: There is significant submesothelial thickening in both SJL and B6 mice after exposure to AdGrem1. The effect is more pronounced in the B6 mice. E: There is also significant increase in submesothelial angiogenesis, which is more prominent in the SJL mice. $\mathbf{F}-\mathbf{H}$ : Gene expression in peritoneal tissues of SJL and B6 mice 7 days after infection with AdGrem1. F and G: Expression of Col1A2 and Vegf is increased in B6 mice, but not in SJL mice. $\mathrm{H}$ : Expression of E-cadherin (Chd1) is decreased in both SJL and B6 mice treated with AdGrem1. $n=5$ per group. Data are expressed as means \pm SD $(\mathbf{D}-\mathbf{H}) .{ }^{*} P<0.05$, ${ }^{* *} P<0.01,{ }^{* * *} P<0.001$. Original magnification: $\times 100($ A and $\mathbf{B})$.
PD, injured mesothelial cells can become denuded from the peritoneal surface or can undergo transition to become myofibroblasts. ${ }^{8}$ There is likely a balance between factors that induce injury and protect mesothelial cells. ${ }^{34}$ The balance between TGF- $\beta$ and BMP7 as agents associated with injury and protection has been investigated previously. ${ }^{12}$ GREM1 was reported to block the expression of BMPs ${ }^{15}$ and to inhibit BMP activity, ${ }^{13}$ which should tip the balance in favor of epithelial transition, and thus may represent a novel injury agent. The role of GREM1 in peritoneal membrane injury has not been previously investigated.

In a stable PD patient population, we were able to demonstrate that GREM1 concentration in the peritoneal effluent was correlated with solute transport. It has been demonstrated that solute transport, as measured by $\mathrm{D} / \mathrm{P}$ creatinine, was a marker of peritoneal membrane angiogenesis, ${ }^{35}$ and that increased peritoneal membrane solute transport was correlated with worse clinical outcomes. ${ }^{7}$ GREM1 appears to be locally produced in the peritoneal cavity, as the peritoneal concentration of GREM1 was significantly higher than was the concentration in the serum. This finding is supported by a recent gene microarray study that found GREM1 expression to be 43-fold greater in the omental tissue compared with subcutaneous tissue. ${ }^{36} \mathrm{We}$ have few useful tools to assess peritoneal membrane function, and few prognostic markers that allow us to identify patients who may be at risk for peritoneal membrane failure or fibrosis. $^{37}$ This observation suggests that GREM1 may be a useful biomarker of peritoneal membrane injury.

We have shown that TGF- $\beta$ appeared to have induced GREM1 expression in B6 mice, so we presume that TGF- $\beta$ may play a role in the up-regulation of GREM1 in PD patients. We did not directly assess this hypothesis in our patient samples. We did observe an increase in GREM1 protein in patients with diabetes, suggesting a role for
A

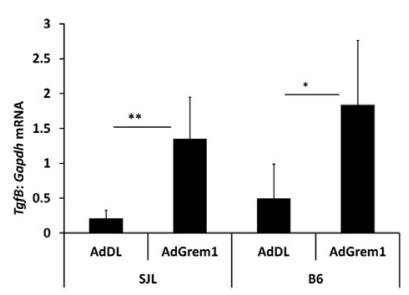

B

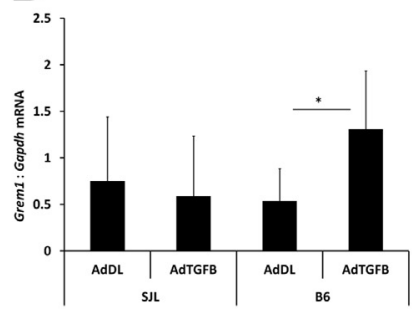

C

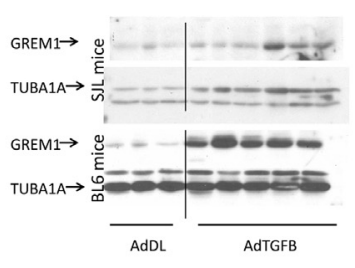

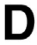

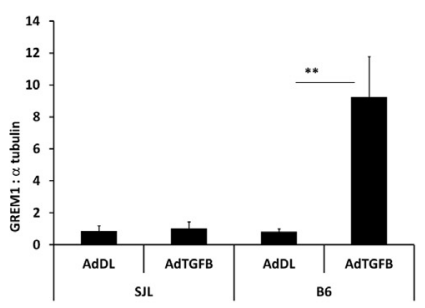

Figure 6 A: AdGrem1 induces Tgfb gene expression in both SJL and BL6 mice as measured by quantitative PCR. B: In mice administered AdTGFB1, only BL6 mice show a significant 2.5-fold increase in Grem1 gene expression. $\mathbf{C}$ and D: Comparison of the protein expression of GREM1 after AdTGFB1 treatment in both BL6 and SJL mice. BL6 mice show a significant increase in GREM1 expression after treatment with AdTGFB1, whereas SJL mice are unresponsive. Representative blot (C) quantified in D. Each lane represents an individual mouse. Band density normalized to alpha-tubulin (TUBA1A). $n=5$ to 6 per group. Data are expressed as means $\pm \mathrm{SD}(\mathbf{A}, \mathbf{B}$, and $\mathbf{D}) .{ }^{*} P<0.05,{ }^{* *} P<0.01$. 
glucose in the regulation of GREM1. Glucose regulation of GREM1 has been observed previously in the setting of diabetic nephropathy. ${ }^{19}$

GREM1 is usually considered an important factor in embryogenesis and to play a role in branching morphogenesis. ${ }^{26}$ More recently, the role of GREM1 in fibrosis ${ }^{18}$ and $\mathrm{EMT}^{38}$ has been explored. We have found that GREM1 may induce fibrosis, angiogenesis, and EMT when overexpressed in the peritoneal tissues of B6 mice. Using dual staining for cytokeratin and $\alpha$ smooth muscle actin, we observed that mesothelial cells in the peritoneum treated with AdGrem1 underwent transition, but that invasion into the submesothelial zone was minimal (Figure 4). Cellular transition and invasion are key features of AdTGFB1induced peritoneal membrane injury. ${ }^{11}$ We hypothesize that the degree of cellular invasion after transition is likely related to the concentration of TGF- $\beta$ to which the mesothelial cells are exposed. In previous work, there was significantly less TGF- $\beta$ expression in the lung after infection with AdGREM1 compared to TGF- $\beta$ expression after AdTGFB $1 .{ }^{18}$ We have previously seen a noninvasive EMT phenotype in the peritoneum of mice after exposure to platelet-derived growth factor B and found that this was associated with a lack of induction of matrix metalloproteinases 2 and $9 .^{29}$

Treatment with BMP7 appears to reverse some of the effects of GREM1. Specifically, rBMP7 treatment appeared to have reversed the GREM1-induced down-regulation of BMP4 and E-cadherin and to have inhibited the GREM1 induction of collagen and VEGF (Figure 3). Some of the histological changes, including submesothelial thickening, angiogenesis, and EMT, did not appear to have been reversed by rBMP7.

Treatment with the pan-TGF- $\beta$ inhibitor 1D11 also appeared to have reversed GREM1-induced downregulation of BMP4 and BMP7 and to have inhibited the GREM1 induction of Colla2 and Vegf (Figure 3). Inhibition of TGF- $\beta$ also apparently prevented GREM1-induced submesothelial thickening and EMT response but did not appear to have fully reversed the angiogenic response to GREM1 (Figure 2).

We previously observed that SJL mice were resistant to TGF- $\beta$-induced fibrosis. ${ }^{32}$ We were intrigued to find that SJL mice had a intense proliferation, EMT, and angiogenic responses to AdGrem1 similar to those in the B6 mice. The angiogenic response was more prominent in the SJL than in the B6 mice (Figure 5E) despite lower expression of Vegf in the SJL mice (Figure 5G). This finding suggests that there are angiogenic pathways independent of Vegf that are induced by GREM1 and that are exaggerated in the SJL mice.

Our observation that GREM1 apparently induced significant peritoneal angiogenesis is a novel finding. Others have shown that GREM1 expression was associated with angiogenesis and was inversely correlated with survival in cancer. ${ }^{39}$ The robust angiogenic response to GREM1 in SJL mice, despite the lack of significant up-regulation of Vegf, along with the lack of suppression of angiogenesis in GREM1 mice treated with 1D11 despite the reduction in $V e g f$ with this therapy, suggest that GREM1 may have direct angiogenic properties not requiring VEGF or TGF- $\beta$. This hypothesis is supported by recent evidence that GREM1 may directly bind the VEGF receptor. ${ }^{20,21}$

In both B6 and SJL mice, TGF- $\beta$ apparently was strongly induced by exposure to GREM1. The complementary regulation of TGF- $\beta$ and GREM1 was observed recently in podocytes exposed to high-glucose conditions ${ }^{40}$ and in renal tubular epithelial cells, ${ }^{41}$ where GREM1 was involved in TGF- $\beta$-induced EMT. On the contrary, it appears as though GREM1 is induced by TGF- $\beta$ in the peritoneum of B6 mice but not SJL mice. Our previous work in animals has suggested that the peritoneal response to injury has a genetic component, ${ }^{32}$ and in patients, gene polymorphisms that are associated with peritoneal membrane function have been identified. ${ }^{42}$ The fibrogenic response to GREM1 in SJL mice suggests that a Greml gene polymorphism may explain the fibrosis resistance in the SJL mice, and may possibly be a genetic determinant of peritoneal membrane function in patients on PD therapy. It is noteworthy that a Greml polymorphism has been recently identified in patients with sarcoidosis, which increases their risk for pulmonary fibrosis sixfold. ${ }^{43}$

In summary, GREM1 appears to induce fibrosis, angiogenesis, and EMT in the peritoneal membrane of mice. These effects appear to be mediated by TGF- $\beta$ and BMP7, although it is not clear whether there is an independent GREM1-induced angiogenic effect. The lack of GREM1 response to TGF- $\beta$ may explain the lack of fibrogenic response previously described in SJL mice and suggests that a Greml gene polymorphism may explain the different peritoneal membrane responses of patients on PD therapy. Finally, we show that GREM1 concentration in PD effluent was correlated with solute transport in PD patients and may be a valuable biomarker of peritoneal membrane health.

\section{References}

1. Vonesh EF, Snyder JJ, Foley RN, Collins AJ: Mortality studies comparing peritoneal dialysis and hemodialysis: what do they tell us? Kidney Int Suppl 2006:S3-S11

2. Khawar O, Kalantar-Zadeh K, Lo WK, Johnson D, Mehrotra R: Is the declining use of long-term peritoneal dialysis justified by outcome data? Clin J Am Soc Nephrol 2007, 2:1317-1328

3. Finkelstein AF, Wuerth D, Finkelstein SH: Quality of life assessments in hemodialysis and peritoneal dialysis patients: an important dimension of patient choice why is the evidence favoring hemodialysis over peritoneal dialysis misleading? Semin Dial 2007, 20:211-213

4. Berger A, Edelsberg J, Inglese GW, Bhattacharyya SK, Oster G: Cost comparison of peritoneal dialysis versus hemodialysis in end-stage renal disease. Am J Manag Care 2009, 15:509-518

5. Williams JD, Craig KJ, Topley N, Von Ruhland C, Fallon M, Newman GR, Mackenzie RK, Williams GT; Peritoneal Biopsy Study Group: Morphologic changes in the peritoneal membrane of patients with renal disease. J Am Soc Nephrol 2002, 13:470-479 
6. Davies SJ, Mushahar L, Yu Z, Lambie M: Determinants of peritoneal membrane function over time. Semin Nephrol 2011, 31:172-182

7. Brimble KS, Walker M, Margetts PJ, Kundhal KK, Rabbat CG: Metaanalysis: peritoneal membrane transport, mortality, and technique failure in peritoneal dialysis. J Am Soc Nephrol 2006, 17:2591-2598

8. Yáñez-Mó M, Lara-Pezzi E, Selgas R, Ramirez-Huesca M, Domínguez-Jiménez C, Jimenez-Heffernan JA, Aguilera A, SánchezTomero JA, Bajo MA, Alvarez V, Castro MA, del Peso G, Cirujeda A, Gamallo C, Sánchez-Madrid F, López-Cabrera M: Peritoneal dialysis and epithelial-to-mesenchymal transition of mesothelial cells [erratum in: N Engl J Med 2005;353:2827]. N Engl J Med 2003, 348:403-413

9. Zhang J, Oh KH, Xu H, Margetts PJ: Vascular endothelial growth factor expression in peritoneal mesothelial cells undergoing transdifferentiation. Perit Dial Int 2008, 28:497-504

10. Aroeira LS, Aguilera A, Selgas R, Ramirez-Huesca M, PerezLozano ML, Cirugeda A, Bajo MA, del Peso G, Sanchez-Tomero JA, Jimenez-Heffernan JA, Lopez-Cabrera M: Mesenchymal conversion of mesothelial cells as a mechanism responsible for high solute transport rate in peritoneal dialysis: role of vascular endothelial growth factor. Am J Kidney Dis 2005, 46:938-948

11. Margetts PJ, Bonniaud P, Liu L, Hoff CM, Holmes CJ, West-Mays JA, Kelly MM: Transient overexpression of TGF- $\{$ beta $\} 1$ induces epithelial mesenchymal transition in the rodent peritoneum. J Am Soc Nephrol 2005, 16:425-436

12. Loureiro J, Schilte M, Aguilera A, Albar-Vizcaíno P, RamírezHuesca M, Pérez-Lozano ML, González-Mateo G, Aroeira LS, Selgas R, Mendoza L, Ortiz A, Ruíz-Ortega M, van den Born J, Beelen RH, López-Cabrera M: BMP-7 blocks mesenchymal conversion of mesothelial cells and prevents peritoneal damage induced by dialysis fluid exposure. Nephrol Dial Transplant 2010, 25:1098-1108

13. Hsu DR, Economides AN, Wang X, Eimon PM, Harland RM: The Xenopus dorsalizing factor Gremlin identifies a novel family of secreted proteins that antagonize BMP activities. Mol Cell 1998, 1: 673-683

14. Costello CM, Cahill E, Martin F, Gaine S, McLoughlin P: Role of gremlin in the lung: development and disease. Am J Respir Cell Mol Biol 2010, 42:517-523

15. Sun J, Zhuang FF, Mullersman JE, Chen H, Robertson EJ, Warburton D, Liu YH, Shi W: BMP4 activation and secretion are negatively regulated by an intracellular gremlin-BMP4 interaction. J Biol Chem 2006, 281:29349-29356

16. Bragdon B, Moseychuk O, Saldanha S, King D, Julian J, Nohe A: Bone morphogenetic proteins: a critical review. Cell Signal 2011, 23: 609-620

17. Michos O, Panman L, Vintersten K, Beier K, Zeller R, Zuniga A: Gremlin-mediated BMP antagonism induces the epithelialmesenchymal feedback signaling controlling metanephric kidney and limb organogenesis. Development 2004, 131:3401-3410

18. Farkas L, Farkas D, Gauldie J, Warburton D, Shi W, Kolb M: Transient overexpression of Gremlin results in epithelial activation and reversible fibrosis in rat lungs. Am J Respir Cell Mol Biol 2011, 44: 870-878

19. Dolan V, Murphy M, Sadlier D, Lappin D, Doran P, Godson C, Martin F, O'Meara Y, Schmid H, Henger A, Kretzler M, Droguett A, Mezzano S, Brady HR: Expression of gremlin, a bone morphogenetic protein antagonist, in human diabetic nephropathy. Am J Kidney Dis 2005, 45:1034-1039

20. Mitola S, Ravelli C, Moroni E, Salvi V, Leali D, Ballmer-Hofer K, Zammataro L, Presta M: Gremlin is a novel agonist of the major proangiogenic receptor VEGFR2. Blood 2010, 116:3677-3680

21. Ravelli C, Mitola S, Corsini M, Presta M: Involvement of $\alpha v \beta 3$ integrin in gremlin-induced angiogenesis. Angiogenesis 2013, 16: 235-243

22. Su WS, Gangji AS, Margetts PM, Bosch J, Yusuf S, Clase CM, Ganame J, Noseworthy M, Lonn E, Jain AK, McCormick B, Brimble KS: The FLUID Study protocol: a randomized controlled study on the effects of bioimpedance analysis and vitamin D on left ventricular mass in peritoneal dialysis patients. Perit Dial Int 2011, 31: $529-536$

23. Twardowski ZJ: Clinical value of standardized equilibration tests in CAPD patients. Blood Purif 1989, 7:95-108

24. Biswas S, Nyman JS, Alvarez J, Chakrabarti A, Ayres A, Sterling J, Edwards J, Rana T, Johnson R, Perrien DS, Lonning S, Shyr Y, Matrisian LM, Mundy GR: Anti-transforming growth factor $ß$ antibody treatment rescues bone loss and prevents breast cancer metastasis to bone. PLoS One 2011, 6:e27090

25. Zeisberg M, Hanai J, Sugimoto H, Mammoto T, Charytan D, Strutz F, Kalluri R: BMP-7 counteracts TGF-beta1-induced epithelial-tomesenchymal transition and reverses chronic renal injury. Nat Med 2003, 9:964-968

26. Shi W, Zhao J, Anderson KD, Warburton D: Gremlin negatively modulates BMP-4 induction of embryonic mouse lung branching morphogenesis. Am J Physiol Lung Cell Mol Physiol 2001, 280: L1030-L1039

27. Sime PJ, Xing Z, Graham FL, Csaky KG, Gauldie J: Adenovectormediated gene transfer of active transforming growth factor-betal induces prolonged severe fibrosis in rat lung. J Clin Invest 1997, 100: 768-776

28. Xing Z, Ohkawara Y, Jordana M, Graham F, Gauldie J: Transfer of granulocyte-macrophage colony-stimulating factor gene to rat lung induces eosinophilia, monocytosis, and fibrotic reactions. J Clin Invest 1996, 97:1102-1110

29. Patel P, West-Mays J, Kolb M, Rodrigues JC, Hoff CM, Margetts PJ: Platelet derived growth factor B and epithelial mesenchymal transition of peritoneal mesothelial cells. Matrix Biol 2010, 29:97-106

30. Sekiguchi Y, Zhang J, Patterson S, Liu L, Hamada C, Tomino Y, Margetts PJ: Rapamycin inhibits transforming growth factor $\beta$-induced peritoneal angiogenesis by blocking the secondary hypoxic response. J Cell Mol Med 2012, 16:1934-1945

31. Wu CF, Chiang WC, Lai CF, Chang FC, Chen YT, Chou YH, Wu TH, Linn GR, Ling H, Wu KD, Tsai TJ, Chen YM, Duffield JS, Lin SL: Transforming growth factor $\beta-1$ stimulates profibrotic epithelial signaling to activate pericyte-myofibroblast transition in obstructive kidney fibrosis. Am J Pathol 2013, 182:118-131

32. Margetts PJ, Hoff C, Liu L, Korstanje R, Walkin L, Summers A, Herrick S, Brenchley P: Transforming growth factor $\beta$-induced peritoneal fibrosis is mouse strain dependent. Nephrol Dial Transplant 2013, 28:2015-2027

33. Dobbie JW: Morphology of the peritoneum in CAPD. Blood Purif 1989, 7:74-85

34. Vargha R, Endemann M, Kratochwill K, Riesenhuber A, Wick N, Krachler AM, Malaga-Dieguez L, Aufricht C: Ex vivo reversal of in vivo transdifferentiation in mesothelial cells grown from peritoneal dialysate effluents. Nephrol Dial Transplant 2006, 21:2943-2947

35. Numata M, Nakayama M, Nimura S, Kawakami M, Lindholm B, Kawaguchi Y: Association between an increased surface area of peritoneal microvessels and a high peritoneal solute transport rate. Perit Dial Int 2003, 23:116-122

36. Hoggard N, Cruickshank M, Moar KM, Bashir S, Mayer CD: Using gene expression to predict differences in the secretome of human omental vs. subcutaneous adipose tissue. Obesity (Silver Spring) 2012, 20:1158-1167

37. Krediet RT, Struijk DG: Peritoneal dialysis membrane evaluation in clinical practice. Contrib Nephrol 2012, 178:232-237

38. Li Y, Wang Z, Wang S, Zhao J, Zhang J, Huang Y: Gremlin-mediated decrease in bone morphogenetic protein signaling promotes aristolochic acid-induced epithelial-to-mesenchymal transition (EMT) in HK-2 cells. Toxicology 2012, 297:68-75

39. Chen MH, Yeh YC, Shyr YM, Jan YH, Chao Y, Li CP, Wang SE, Tzeng CH, Chang PM, Liu CY, Chen MH, Hsiao M, Huang CY: Expression of gremlin 1 correlates with increased angiogenesis and progression-free survival in patients with pancreatic neuroendocrine tumors. J Gastroenterol 2013, 48:101-108 
40. Li G, Li Y, Liu S, Shi Y, Chi Y, Liu G, Shan T: Gremlin aggravates hyperglycemia-induced podocyte injury by a TGF $\beta /$ smad dependent signaling pathway. J Cell Biochem 2013, 114: 2101-2113

41. Rodrigues-Diez R, Lavoz C, Carvajal G, Rayego-Mateos S, Rodrigues Diez RR, Ortiz A, Egido J, Mezzano S, Ruiz-Ortega M: Gremlin is a downstream profibrotic mediator of transforming growth factor-beta in cultured renal cells. Nephron Exp Nephrol 2012, 122:62-74
42. Gillerot G, Goffin E, Michel C, Evenepoel P, Biesen WV, Tintillier M, Stenvinkel P, Heimburger O, Lindholm B, Nordfors L, Robert A, Devuyst O: Genetic and clinical factors influence the baseline permeability of the peritoneal membrane. Kidney Int 2005, 67:2477-2487

43. Heron M, van Moorsel CH, Grutters JC, Huizinga TW, van der Helmvan Mil AH, Nagtegaal MM, Ruven HJ, van den Bosch JM: Genetic variation in GREM1 is a risk factor for fibrosis in pulmonary sarcoidosis [erratum in: Tissue Antigens 2011;77:365]. Tissue Antigens 2011, 77:112-117 